\title{
General solutions of the Monge-Ampère equation in $n$-dimensional space
}

\author{
D.B. Fairlie \\ Department of Mathematical Sciences \\ University of Durham, Durham DH1 3LE \\ and A.N. Leznov \\ Institute for High Energy Physics, 142284 Protvino, \\ Moscow Region, Russia
}

August 3, 2018

\begin{abstract}
It is shown that the general solution of a homogeneous MongeAmpère equation in $n$-dimensional space is closely connected with the exactly (but only implicitly) integrable system

$$
\frac{\partial \xi_{j}}{\partial x_{0}}+\sum_{k=1}^{n-1} \xi_{k} \frac{\partial \xi_{j}}{\partial x_{k}}=0
$$

Using the explicit form of solution of this system it is possible to construct the general solution of the Monge-Ampère equation.
\end{abstract}




\section{Introduction}

The original form of Monge-Ampère equation in $n$-dimensional space is the following one [1, 2, 3]

$$
\operatorname{det}\left(\phi_{\rho, \sigma}\right)=F\left(\phi, \phi_{\rho}, x_{\rho}\right) \quad(0 \geq \rho, \sigma \geq n-1)
$$

where $\phi_{\rho, \sigma} \equiv \frac{\partial^{2} \phi}{\partial x_{\rho} \partial x_{\sigma}}$ and $x_{\rho}$ are the coordinates of the space.

With respect to each of its independent coordinates (2) is an equation of second order and consequently its general solution must depend on two arbitrary functions of $n-1$ independent arguments. We shall show that the homogeneous form of this equation, i.e the special case where the function $F \equiv 0$, is exactly integrable. In this case the equation corresponds to the condition that a hypersurface in $\mathbb{R}^{n+1}$ has zero Gaussian Curvature. In what follows we shall refer to this equation with $F=0$ as the Monge-Ampère equation. There is a deep connection between this equation and the equation

$$
\operatorname{det}\left|\begin{array}{cc}
k \phi & \phi_{j} \\
\phi_{i} & \phi_{i j}
\end{array}\right|=0 .
$$

For all $k \neq 0$ this equation after a trivial change of unknown function $\left(\phi^{\frac{k}{k-1}}\right.$ if $k \neq 1,0, \log \phi$ if $k=1$ ) coincides with the Monge Ampère equation. The particular case $k=0$ corresponds to the Universal Field Equation [7] whose general solution was found by means of a Legendre Transform in [6]. This solution is connected with the solution of the Monge Ampère equation in $n+1$ dimensional space by the substitution

$$
\Phi_{M A}\left(x_{1}, x_{2}, \ldots x_{n+1}\right)=x_{n+1} \phi_{U F E}\left(x_{1}, x_{2}, \ldots x_{n}\right)
$$

Its solution by the methods of the present paper will be given elsewhere.

\section{The general construction}

The vanishing of the determinant means that the rows (or columns) of its $n \times n$ matrix are linearly dependent.

$$
\sum_{\sigma} \phi_{\rho, \sigma} \alpha_{\sigma}=0
$$


where $\alpha_{\sigma}$ are some functions of the coordinates $x_{\rho}$. From this observation it is easy to see that if $\phi$ is homogeneous of weight one, i.e. $\phi\left(\lambda x_{\rho}\right)=\lambda \phi\left(x_{\rho}\right)$ then by Euler's theorem on homogeneous functions

$$
\sum_{\sigma} x_{\sigma} \frac{\partial \phi}{\partial x_{\sigma}}-\phi=0
$$

Differentiation of this equation with respect to $x_{\rho}$ leads to an equation of the form (5) and thus implies that any homogeneous function of weight one satisfies the Monge-Ampère equation. Thus one class of solutions is easily obtained.

Let us rewrite (5) in equivalent form

$$
\frac{\partial \mathcal{R}}{\partial x_{\rho}} \equiv \frac{\partial}{\partial x_{\rho}} \sum_{\sigma} \phi_{\sigma} \alpha_{\sigma}=\sum_{\sigma} \phi_{\sigma} \frac{\partial \alpha_{\sigma}}{\partial x_{\rho}}
$$

and will consider $\mathcal{R}$ as a function of $n$ independent variables $\alpha_{\rho}$ assuming that $\left.\operatorname{det}\left(\frac{\partial \alpha_{\sigma}}{\partial x_{\rho}}\right) \neq 1\right)$.

From (17) we obtain immediately

$$
\phi_{\sigma}=\frac{\partial \mathcal{R}}{\partial \alpha_{\sigma}}, \quad \mathcal{R}=\sum \alpha_{\sigma} \frac{\partial \mathcal{R}}{\partial \alpha_{\sigma}}
$$

or that $\mathcal{R}$ is a homogeneous function of variables $\alpha_{\rho}$ of degree one. So

$$
\mathcal{R}=\alpha_{0} R\left(\frac{\alpha_{j}}{\alpha_{0}}\right), \quad \phi_{0}=R-\sum \xi_{j} \frac{\partial R}{\partial \xi_{j}}, \quad \phi_{j}=\frac{\partial R}{\partial \xi_{j}}
$$

here $\xi_{j}=\frac{\alpha_{j}}{\alpha_{0}}$ and from now on all Latin indices take values from 1 up to $n-1$.

The condition of compatibility of (8) - the equivalence of the second mixed derivatives - will give us the dependenced of the $n-1$ new variables $\xi_{j}$ upon the $n$ previous variables $x_{\rho}$.

We have firstly

$$
\frac{\partial}{\partial x_{k}} \frac{\partial R}{\partial \xi_{j}}=\frac{\partial}{\partial x_{j}} \frac{\partial R}{\partial \xi_{k}}
$$

and secondly

$$
\frac{\partial}{\partial x_{k}} \frac{\partial \phi}{\partial x_{0}}=-\sum \xi_{j} \frac{\partial}{\partial x_{k}} \frac{\partial R}{\partial \xi_{j}}=-\sum \xi_{j} \frac{\partial}{\partial x_{j}} \frac{\partial R}{\partial \xi_{k}}=\frac{\partial}{\partial x_{0}} \frac{\partial R}{\partial \xi_{k}}
$$


From the last equality $(10)$ we readily obtain

$$
\sum_{k}\left(\frac{\partial \xi_{k}}{\partial x_{0}}+\sum_{r=1}^{n-1} \xi_{r} \frac{\partial \xi_{k}}{\partial x_{r}}\right) \frac{\partial^{2} R}{\partial \xi_{k} \partial \xi_{j}}=0
$$

With respect to the variables $q_{k}=\frac{\partial \xi_{k}}{\partial x_{0}}+\sum_{r=1}^{n-1} \xi_{r} \frac{\partial \xi_{k}}{\partial x_{r}}$ (11) is a linear system of algebraic equations which it is possible to solve in the two cases given by the Fredholm alternative;

$$
\operatorname{det}_{n-1}\left(\frac{\partial^{2} R}{\partial \xi_{k} \partial \xi_{j}}\right)=0, \quad q_{k} \neq 0 ; \quad \operatorname{det}_{n-1}\left(\frac{\partial^{2} R}{\partial \xi_{k} \partial \xi_{j}}\right) \neq 0, \quad q_{k}=0
$$

We shall consider in this paper the second possibility, hoping to come back to the first one in futher publications and restrict ourselves now only to the simplest nontrivial example $n=3$ in section 5 .

\section{Solution of equations of hydrodynamic type}

The system of equations

$$
\frac{\partial \xi_{k}}{\partial x_{0}}+\sum_{k=1}^{n-1} \xi_{k} \frac{\partial \xi_{k}}{\partial x_{k}}=0
$$

from the physical point of view is that of the equations of velocity flow in hydrodynamics in $n-1$ dimensional space.

In the simplest case $n=2$ this is the one component equation

$$
\frac{\partial \xi}{\partial x_{0}}+\xi \frac{\partial \xi}{\partial x_{1}}=0
$$

which is connected with the name of Monge who first discovered its general solution in implicit form

$$
x_{1}-\xi x_{0}=f(\xi)
$$

where $f(\xi)$ is an arbitrary function of its argument.

The generalisation of this result to the multidimensional case (14) was found by D.B.Fairlie [0] and consists in the following construction. Suppose that the system of $n-1$ equations

$$
x_{j}-\xi_{j} x_{0}=Q^{j}\left(\xi_{1}, \xi_{2}, \ldots \xi_{n-1}\right)
$$


is solved for the unknown functions $\xi_{j}$. Each solution of this system (15) will satisfy (13).

This may be proved as follows. After differentiation of each equation of (15) with respect to the variables $x_{k}$ we obtain

$$
\delta_{k, j}=\sum\left(\frac{\partial Q^{j}}{\partial x_{k}}+\delta_{j, k}\right) \frac{\partial \xi_{k}}{\partial x_{k}}
$$

or

$$
\frac{\partial \xi_{j}}{\partial x_{k}}=\left(I x_{0}+\frac{\partial Q}{\partial \xi}\right)_{j, k}^{-1}
$$

The result of differentiation of (15) with respect to $x_{0}$ has as a corollary

$$
-\frac{\partial \xi_{j}}{\partial x_{0}}=\left[\left(I x_{0}+\frac{\partial Q}{\partial \xi}\right)^{-1} \xi\right)_{j}
$$

The comparision of (17) and (18) proves that system (13) is satisfied.

\section{Continuation.}

Up to now we have used only the condition of compatibility (10). Now let us return to equations (9). By simple computations using the explicit form for derivatives (17) it is easy to show that equations (9) may be written in the form

$$
\sum \frac{\partial}{\partial \xi_{k}} Q^{r} \frac{\partial^{2} R}{\partial \xi_{r} \partial \xi_{j}}=\sum \frac{\partial}{\partial \xi_{j}} Q^{j} \frac{\partial^{2} R}{\partial \xi_{r} \partial \xi_{k}} .
$$

or

$$
\sum \frac{\partial^{2} R}{\partial \xi_{r} \partial \xi_{j}} Q^{j}=\frac{\partial L}{\partial \xi_{r}}
$$

where $L$ is an arbitrary function of $n-1$ arguments $\xi_{k}$. With respect to the functions $Q^{j}(20)$ is a linear system of algebraic equations the matrix of which has the determinant not equal to zero ( the second case of the (13).

The solution of (20) in terms of Cramers determinants has the usual form

$$
Q^{j}=\left[\left(\frac{\partial^{2} R}{\partial \xi \partial \xi}\right)^{-1} \frac{\partial L}{\partial \xi}\right]^{j}
$$


and so all of the functions $Q^{j}$ are represented in terms of only two arbitrary functions $R, L$ and their derivatives up to the second and first orders respectively.

So with the help of equations (8) whose integrability is ensured by the above analysis and (15) we obtain the solution of the Monge-Ampère equation which depends upon two arbitrary functions each of $n-1$ independent arguments and which fulfils the claim of the introduction to provide the general solution for the equation under consideration.

\section{The simplest examples}

\section{$5.1 n=2$}

From (8) we obtain

$$
\frac{\partial \phi}{\partial x_{0}}=R-\xi_{1} \frac{\partial R}{\partial \xi_{1}}, \quad \frac{\partial \phi}{\partial x_{1}}=\frac{\partial R}{\partial \xi_{1}}
$$

The equation (14) and its solution are as follows;

$$
\frac{\partial \xi_{1}}{\partial x_{0}}+\xi_{1} \frac{\partial \xi_{1}}{\partial x_{1}}=0, \quad x_{1}-\xi_{1} x_{0}=F\left(\xi_{1}\right)
$$

In this case the conditions (8) are absent and general solution of the MongeAmpère equation is determined by the pair of arbitrary functions $R, F$ each of one argument $\xi_{1}$.

\section{$5.2 n=3$ - the general case}

$$
\frac{\partial \phi}{\partial x_{0}}=R-\xi_{1} \frac{\partial R}{\partial \xi_{1}}-\xi_{2} \frac{\partial R}{\partial \xi_{2}}, \quad \frac{\partial \phi}{\partial x_{1}}=\frac{\partial R}{\partial \xi_{1}}, \quad \frac{\partial \phi}{\partial x_{2}}=\frac{\partial R}{\partial \xi_{2}}
$$

The connection between the arguments $\xi$ and $x$ keeping in mind (21) takes the form

$$
\begin{aligned}
& x_{1}-\xi_{1} x_{0}=Q^{1}=\frac{R_{22} L_{1}-R_{12} L_{2}}{R_{22} R_{11}-R_{12} R_{21}} \\
& x_{2}-\xi_{2} x_{0}=Q^{2}=\frac{-R_{12} L_{1}+R_{22} L_{2}}{R_{22} R_{11}-R_{12} R_{21}}
\end{aligned}
$$

where $L_{1} \equiv \frac{\partial L}{\partial \xi_{1}}$ and so on. 


\section{$5.3 n=3$-the degenerate case}

The first possibility in (13) means $\operatorname{Det}_{2}\left(\frac{\partial^{2} R}{\partial \xi} \partial \xi\right)=0$. This exactly the MongeAmpère equation of first subsection with the solution

$$
\frac{\partial R}{\partial \xi_{2}}=P-q \frac{\partial P}{\partial q}, \quad \frac{\partial R}{\partial \xi_{1}}=\frac{\partial P}{\partial q}, \quad \xi_{1}-q \xi_{1}=F(q)
$$

Let us substitute all these expressions into (24). We obtain

$$
\frac{\partial \phi}{\partial x_{0}}=R-\left(\xi_{1}-\xi_{2} q\right) \frac{\partial P}{\partial q}-\xi_{2} P, \quad \frac{\partial \phi}{\partial x_{2}}=P-q \frac{\partial P}{\partial q}, \quad \frac{\partial \phi}{\partial x_{1}}=\frac{\partial P}{\partial q}
$$

The condition of compatibility ( the equality of a second mixed derivatives) gives the equations

$$
q_{x_{2}}=-q q_{x_{1}}, \quad q_{x_{0}}=-F(q) q_{x_{1}}, \quad q q_{x_{3}}=-F(q) q_{x_{2}}
$$

which are compatible and have the common solution

$$
-x_{1}+q x_{2}-F(q) x_{0}=\Phi(q)
$$

where $\Phi(q)$ is an arbitrary function of its argument.

We see that the degenerate solution of the Monge-Ampère equation is determined in this case by three arbitrary functions of one argument.

\section{Conclusion remarks}

The general solution of the the Monge-Ampère equation constructed here reminds one of the situation with regard to linear ordinary second order equations when one solution is known; there is a well known procedure for the construction of a first order differential equation whose solution yields the general solution of the original equation. Here something of the same nature is discovered; an arbitrary homogeneous function of weight one satisfies the Monge Ampère equation automatically. We then construct a set of first order equations which are integrable, though only implicitly. Their solution then implies the general solution to our original equation. This construction suggests that equations with the property that an any homogeneous function of given degree is a solution may be also solved by this method, for example the Universal field equation introduced in [4] [5] whose 3 dimensional version describes developable surfaces and which admits homogeneous solutions of degree zero. We intend to return to this matter in a subsequent paper. 


\section{References}

[1] Zwillinger D. Handbook of Differential Equations Academic Press (1989)

[2] Bedford E. and Taylor B.A. Inventiones 37 (1976) 1-44.

[3] Progorelov A.V. Soviet Math (Doklady) 12 (1971) 1193 and 1727.

[4] Fairlie D.B. Govaerts J. and Morozov A., Nucl. Phys. B373 (1992) 214232.

[5] Fairlie D.B. and Govaerts J.Journal of Mathematical Physics.33 (1992) 3543-3566.

[6] Fairlie D.B. and Govaerts J.J. Phys A (1993) 3339-3347.

[7] D.B. Fairlie, Equations of Hydrodynamic Type (1993) DTP/93/31 